\title{
From Visual Behavior to Signage Design: A Wayfinding Experiment with Eye-Tracking in Satellite Terminal of PVG Airport
}

\author{
Chengyu Sun, Shuyang Li ${ }^{(凶)}$, Yinshan Lin, and Weilin Hu \\ Tongji University, Yangpu 200092, Shanghai, China
}

\begin{abstract}
Passengers principally rely on signage to making wayfinding decisions in transportation buildings. Most existing research focuses on the analysis of the wayfinding trajectory, but there is less attention on the process of how passengers make the wayfinding decision. So, it is hard to accurately locate the causes of the wrong wayfinding decision. Taking the Satellite Terminal of Shanghai Pudong International Airport (PVG Airport) as an example, we adopted the eye-tracking technology and recorded the eye-tracking data of passengers observing the signage and making wayfinding decisions. Then, we compared and analyzed the data, presenting it by data visualization. This study found the causes of passengers making wrong wayfinding decisions and the visual behavior of wayfinding: the reconfirmation behavior, the priority of attention, and the clockwise observation. Finally, corresponding suggestions for signage design optimization are put forward regarding some wayfinding decision points. As a result, the optimized signage system in the satellite terminal is welcomed by the passengers two months later according to monthly questionnaires.
\end{abstract}

Keywords: Signage design · Data visualization · Visual behavior .

Eye-tracking · Wayfinding · Transportation building

\section{Introduction}

Transportation building is an important type of architecture in wayfinding research. Passengers usually have a clear wayfinding purpose in transportation buildings, so it is necessary to design a perfected signage system to improve the wayfinding efficiency.

Wayfinding is a process from environmental perception to decision-making. Eyetracking technology is used to record this wayfinding process, and the specific causes of the wrong wayfinding decision can be accurately located by analyzed the eye-tracking data. We proposed a data visualization Based on the HSB color code to analyzing the relationship between the interior space, the signages, and the wayfinding behavior. In this way, we can intuitively see the gaze paths and observation areas of various passengers, which is convenient for concluding the visual behavior of wayfinding. These visual behaviors can provide designers with more references when designing a signage system. 


\section{Background}

The Satellite Terminal of Shanghai Pudong International Airport (Hereinafter PVG Airport) is a typical mega-scale transportation building with 134 boarding gates and approximately 3,600 signboards. The construction area of the Satellite Terminal of PVG airport is about $670,000 \mathrm{~m}^{2}$. Inside the building, the interior space along the walking path is extremely similar. Besides, there are intricate passenger paths in the Satellite Terminal of PVG airport, with paths from entrances to gates taking 30 minutes on average for passengers.

Considering that the unaffordable cost and the difficulty of an on-site wayfinding experiment, a virtual wayfinding experiment platform is developed. Based on the method of "high-resolution panorama + low level of detailed 3D model", the interior space of the Satellite Terminal of PVG airport is reproduced as a virtual reality scene (Fig. 1). Participants were assigned wayfinding tasks randomly, and their wayfinding trajectories were recorded by the program. As a result, a total of 3382 virtual wayfinding experiments (175 people) were completed in three weeks.

Through the analysis of the wayfinding trajectory (Fig. 2), we can quickly find at which wayfinding decision points participants make wrong decisions. However, the trajectory is still not enough to explain why the participants make wrong decisions at this wayfinding decision point. Therefore, it is necessary to design a further experiment to explore the specific causes of the wrong decision.

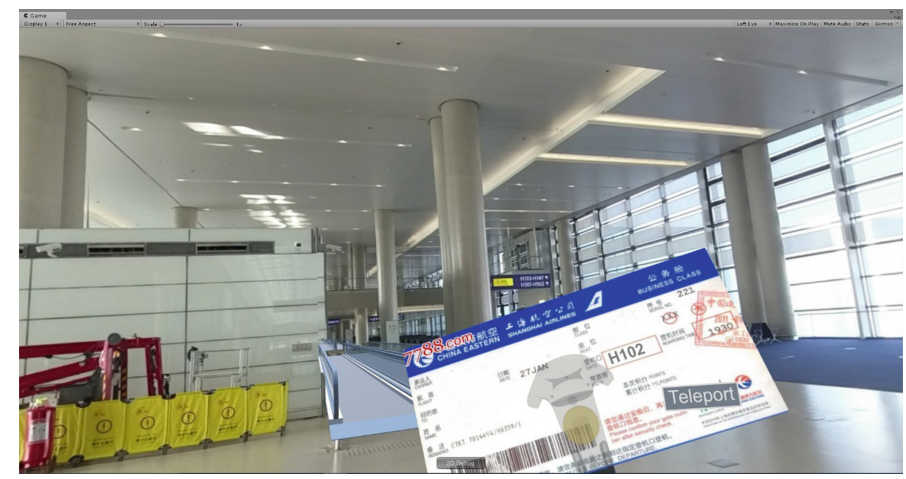

Fig. 1. Virtual wayfinding experiment platform 


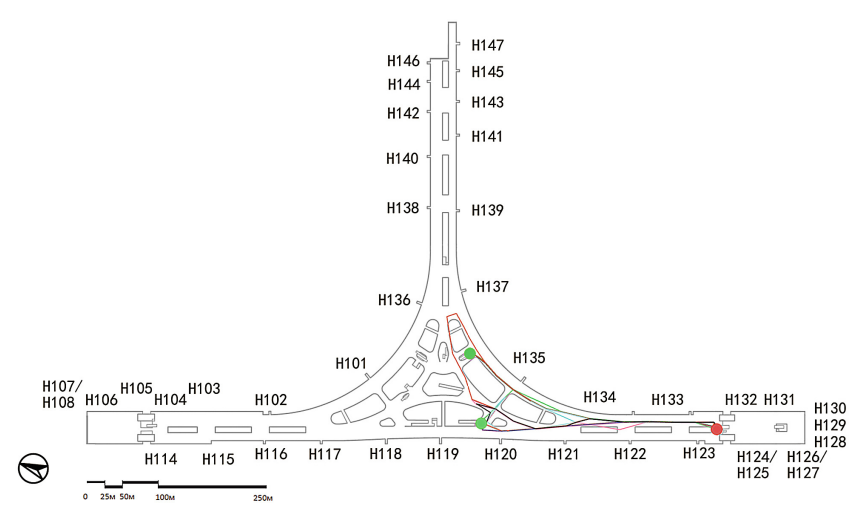

Fig. 2. Trajectories of virtual wayfinding experiment

\section{The Necessity of Exploring Visual Behavior}

Wayfinding behavior is related to human cognition. In transportation buildings, passengers need to find the destination quickly and accurately, so the signage system is remarkably influential (Arthur and Passini 1992). Tzeng and Huang (2009) discovered that signages play a more important role in the enclosed space and the open L-shaped space. Xu, et al. (2010) studied the impact of the distance, position, and color of signboards on the efficiency of wayfinding. They concluded that the signboards at the exit and the turning point are the most concerned and the passengers will follow the direction of the signboards again and again even though it is wrong.

The above studies showcase the relationship between signages and users. While, designers tend to ignore passengers' wayfinding behavior when they design the signage system and wrongly assumed that wayfinding is synonymous with signage (Carpman and Grant 2002). As a result, the signage system could not satisfy users.

Through the eye-tracking technology, passengers' visual behavior can be recorded, which provides an opportunity to study how passengers observe the surrounding environment and how they make wayfinding decisions (Till and Babcock 2011). SchromFeiertag, et al. (2017) developed a virtual wayfinding experiment platform with eyetracking technology, to explore the attention maps and objects of interest for passengers. Wiener, et al. (2012) took a series of eye-tracking experiments to survey visual behavior and decision making in wayfinding. They found that participants tend to choose the path with a longer line of sight and the gaze bias effects are a general phenomenon.

From the eye-tracking data, we can specifically analyze the visual behavior of wayfinding in a more targeted way, so that it can support the melioration of signage system design and interior design.

\section{On-site Wayfinding Experiment with Eye-Tracking}

\subsection{Setting and Participants}

In this experiment, we selected 8 wayfinding decision points where participants made wrong decisions frequently in the virtual experiment as a trial group. 2 wayfinding 
decision points with no record of decision error were added as a control group. The red fan-shaped area (Fig. 3) represents the initial viewing angle of the participants before the experiment started. There are 8 wayfinding tasks on each wayfinding decision point, including the search for the boarding gate, VIP lounge, currency exchange, etc. 80 wayfinding tasks in total.

8 people participated in this experiment between 18 and 46 years old, with an approximately equal number of men and women. All the participants had the experience of taking the airplane and knew the boarding process, but they had never been to the Satellite Terminal of PVG airport. Participants were equipped with a mobile eye-tracking device (Dikablis Glass 3) in this experiment. This device has three independent cameras: front camera for what the wearer is observing, and two cameras for eye movements of the wearer.

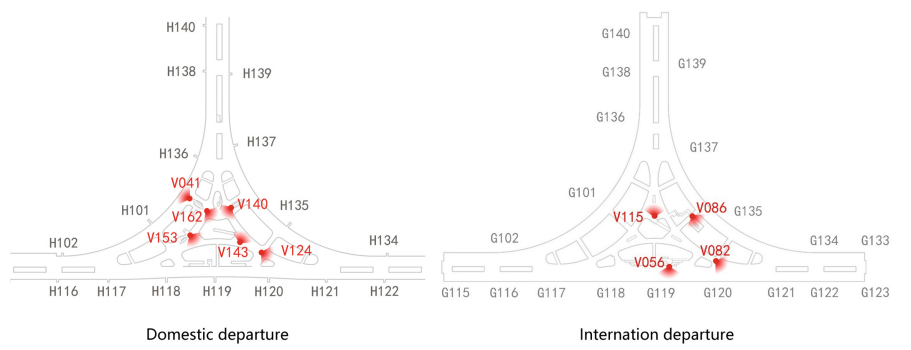

Fig. 3. Wayfinding decision points of the on-site experiment

\subsection{Procedure}

In order to eliminate the influence of sunlight, this experiment was carried out at night with sufficient and stable lighting. The participants corrected the eye-tracking device with the help of the experimenter, and aimlessly observed for 3 minutes to adapt to this state. Then, the experimenter brought the participants to the wayfinding decision point and randomly selected a wayfinding task. After the experimenters having explained the wayfinding task, the participants began to observe the surrounding environment and signboards. Participants informed the experimenter orally when they made a wayfinding decision. Finally, the experimenter interviewed and recorded the feelings and doubts of the participants when they were trying to find the way (Fig. 4).

\subsection{Data Analysis}

\subsubsection{Data Visualization of Single Wayfinding Task}

Eye-tracking data is shown on the panorama of the wayfinding decision point. The fixation duration is presented as a heatmap (observation more than $0.2 \mathrm{~s}$ was considered as fixation). The gaze path is based on the time sequence of the fixation area.

We divide different observation areas and display the eye-tracking data on each observation area respectively. The fixation area and gaze path will change when the 

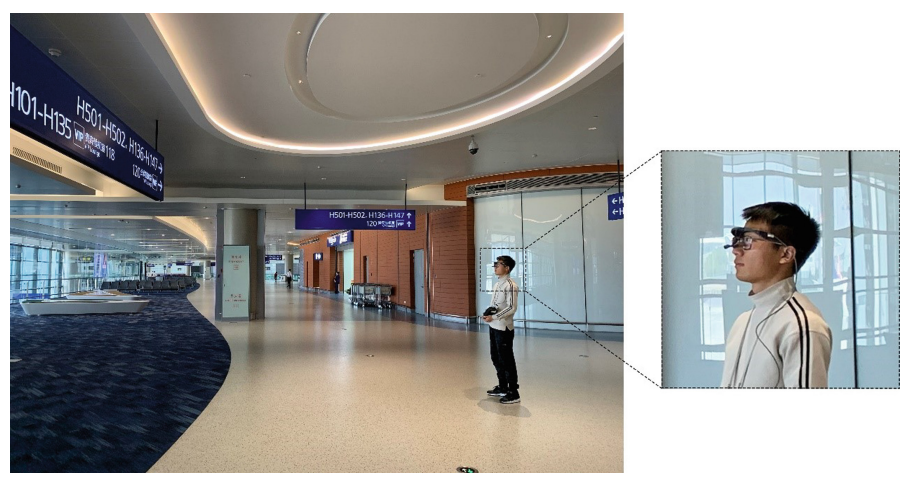

Fig. 4. Participant in the on-site wayfinding experiment with mobile eye-tracking

turning head occurs, so different observation areas should be differentiated for more accurate analysis. The observation areas are also sorted by the time sequence, and the order is represented by red numbers (Fig. 5).

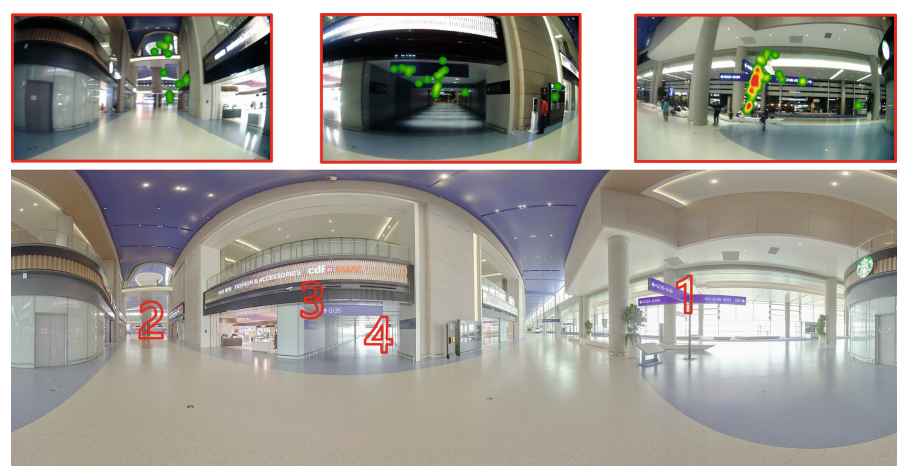

Fig. 5. Data visualization of single wayfinding task

\subsubsection{Data Visualization Based on the HSB Color Code}

Taking a wayfinding decision point as the research object, we try to find the common rules of the visual behavior when participants searching for the wayfinding information at this decision point. A coordinate system is established in the panorama of the wayfinding decision point, and the region of interest is coded in the form of coordinate points. The horizontal axis of the coordinate system is divided into $360^{\circ}$. The vertical axis of the coordinate system is divided into $180^{\circ}$ and the sightline as high as the height $(1.65 \mathrm{~m}$ in general) as $0^{\circ}$.

HSB (Hue-Saturation-Brightness) colors were used to label the interest area for participants and we define that the horizontal coordinate as hue value and the vertical coordinate as the brightness value. The depth of space can be represented by the saturation 
value, but this study does not involve the depth now. To make the color label more intuitive, we cut out a part of the whole chromatography as the new chromatography, in accordance with the horizontal axis of the coordinate system. The color in the bar graph corresponds to the HSB color and represents the region of interest, the horizontal axis of the bar chart represents the proportion of fixation duration. Considering that the fixation duration of each participant is different (from 4 to 30 seconds), we pay more attention to how the participants allocate the observation time, so the proportion of fixation duration is better more informing than the absolute value. Meanwhile, Fixation duration presented on the building layout, the radius of the sector represents the cumulative value of the 8 participants' observation time.

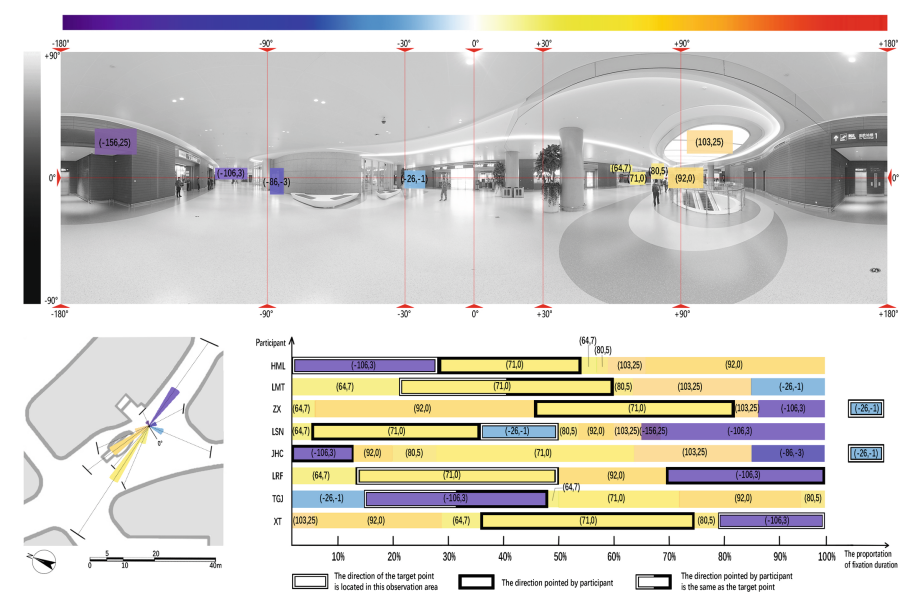

Fig. 6. Data visualization of wayfinding experiments at V153 wayfinding decision point

\subsection{Results}

\subsubsection{Visual Behavior of Wayfinding}

- The Reconfirmation Behavior

To save energy and get to the boarding gate in time, participants appear to have a reconfirmation behavior when they search the wayfinding information in this huge interior space. When they notice the information of the target place on a signboard nearby, participants will then follow the pointed direction and search for the information of the target on the next distant signboard (Fig. 5). If they can't find it, they will feel anxious, leading them to make mistakes in wayfinding.

- The Priority of Attention

Such special places like the escalator, doorway, and other places where building space changes, passengers subconsciously think that there should be signboards or other important information (Fig. 6). Meanwhile, passengers will ignore the narrow passage, or they are unwilling to choose such a path. 
Color and luminescence are also important factors to the priority of attention. The lamps, the LED electronic display screen, and the luminous shop front are easier to attract the attention of the participants than the signboards. When having observed the signboard (the color of the panel is blue), the participants were first attracted to the yellow fonts rather than the white fonts.

- The Clockwise Observation

When there is no signage in front of the visual field or no needed information on the signboards, the participants usually observe along the clockwise direction (right side). In the bar chart of Fig. 7, the color of each line changes from yellow to orange, and then to purple and blue. It means that most people turn to the right and search for information, which may be related to the fact that most of the participants are right-handed. While a left-handed participant always observes in a counterclockwise direction.

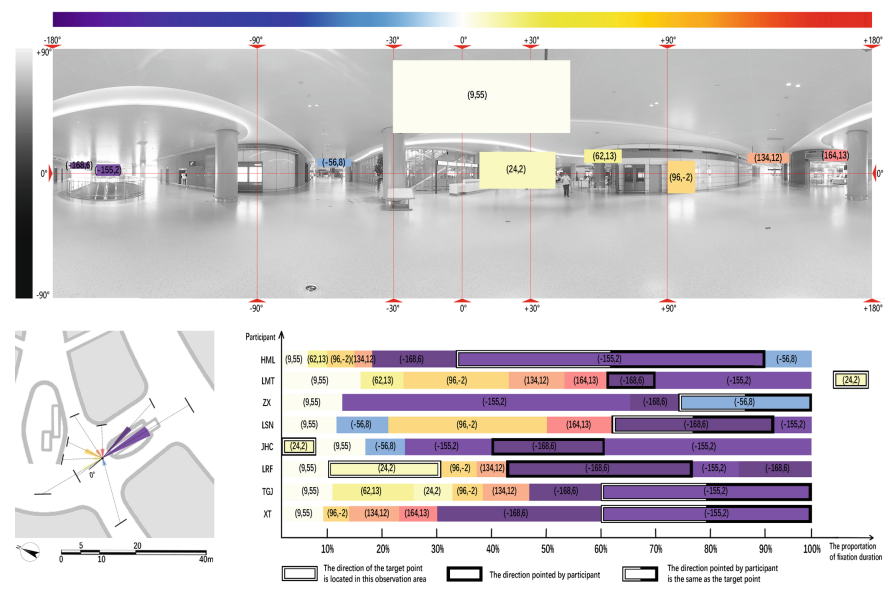

Fig. 7. Data visualization of wayfinding experiments at V140 wayfinding decision point

\subsubsection{The Causes of Making Wrong Wayfinding Decisions}

Combined with the oral statements and eye-tracking data of the participants, various ingrained causes of the wayfinding problems are analyzed to guide subsequent design optimization (Table 1):

\section{Optimization of Signage Design}

\subsection{Guidelines of Optimization}

After sorting out the results of the wayfinding experiment, we propose a guideline for optimizing the signage design: 
Table 1. Causes of wayfinding problems

\begin{tabular}{l|l}
\hline Wayfinding decision point & Causes \\
\hline V041 & Some wayfinding information guide to the detour path \\
\hline V056 & $\begin{array}{l}\text { Wayfinding information for the same destination while } \\
\text { pointing to different directions }\end{array}$ \\
\hline V140 & $\begin{array}{l}\text { There is no signboard at this crossroads and wayfinding } \\
\text { information guide to the detour path }\end{array}$ \\
\hline V082, V086, V124, V153, V162 & Insufficient wayfinding information on signboards \\
\hline
\end{tabular}

- At the starting point of each floor, all directions should be marked on the signboards, and a wayfinding map should be placed here for passengers.

- Signboards should be added where the building space changes.

- At the multi-directional wayfinding decision point, signboards should be placed in all directions, and redundant and ambiguous information should be removed.

- Adjust the position and the direction of the signboards according to the observation angle from the passenger's path.

- Distinguish the information of different wayfinding processes (departure, arrival, transit, etc.) by different colors.

- The contents of the signboards should follow a hierarchy (using different sizes of font and sign) according to the priorities.

\subsection{Cases of Optimization}

\subsubsection{Set Signboards at Specific Positions}

If the sightline is blocked that passengers can't see the signboards in the distance when the interior space changes, the signboards should be set at these positions. At the V153 decision point, two redundant signboards are removed and two signboards are added to ensure the continuity of observation (Fig. 8).

\subsubsection{Adjust Wayfinding Information Based on the Reconfirmation Behavior}

The wayfinding information on the signboards should be coherent to ensure the information can echo with each other. As shown in Fig. 9, four redundant signboards are removed, and a crossroad signboard (directing to three directions) is added. All the wayfinding information appeared twice, on the hanging signboards and crossroads signboard, so passengers can easily confirm.

\subsubsection{Adjust the Content by Priority}

Process wayfinding information (Departure, arrival, boarding gate) is the most important, followed by information of the functional facilities (restaurant, shop, toilet). We can differentiate wayfinding information by designing it as luminous or not, in colors and sizes (Fig. 10). 


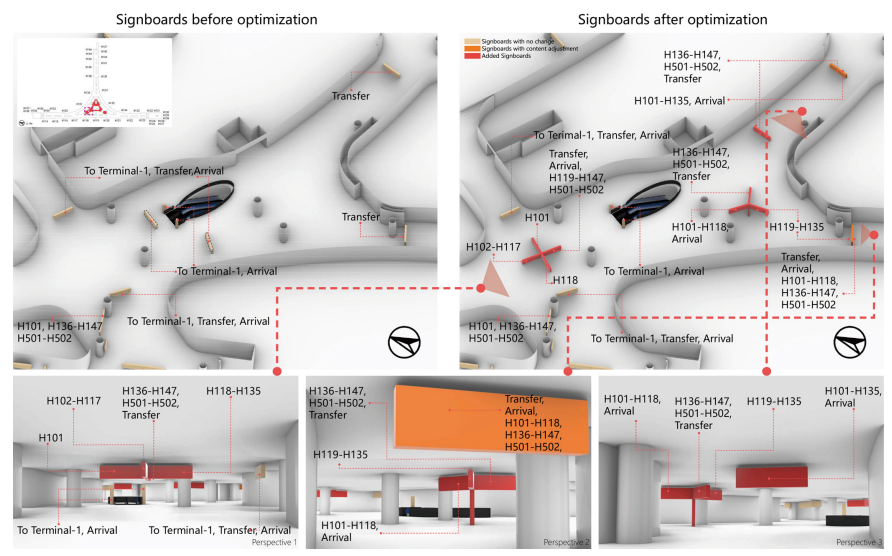

Fig. 8. Optimization of signage design at V153 wayfinding decision point

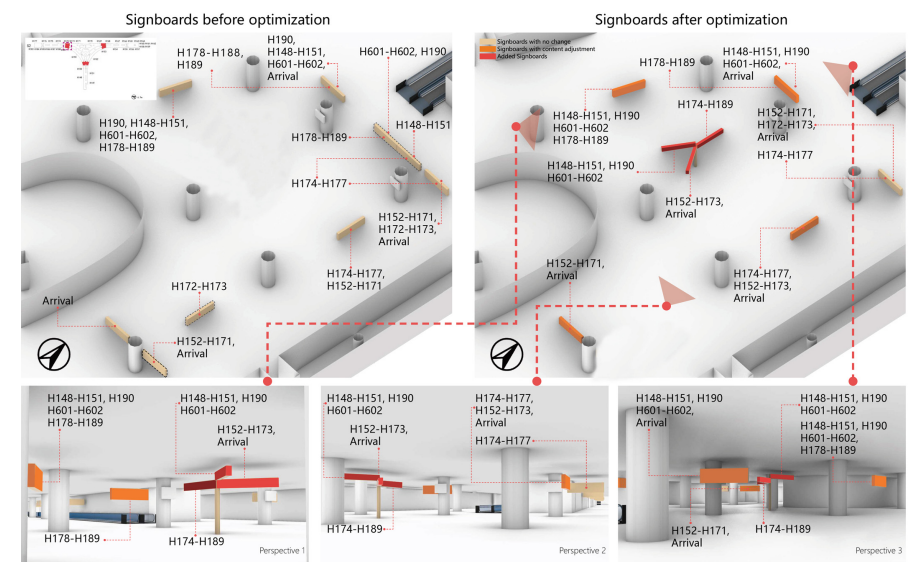

Fig. 9. Optimization of signage design based on the reconfirmation behavior

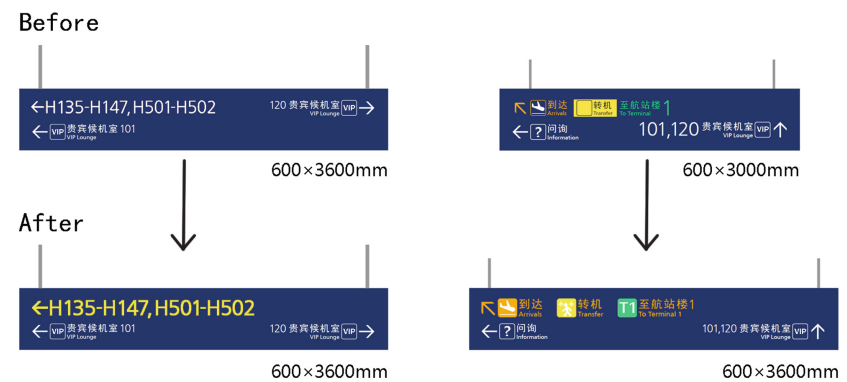

Fig. 10. Optimization of signboards design 


\section{Summary}

As a powerful supplement to conventional wayfinding research, eye-tracking technology has great potential. Taking the Satellite Terminal of PVG Airport as an example, the eye-tracking data can be visualized on the panorama of wayfinding decision points. Therefore, the specific causes of the wrong wayfinding decision can be accurately located. Finally, suggestions for signage design optimization are put forward in some wayfinding decision points. It is estimated that the optimized project will take 9 months to complete which start in October 2020. After that, a systematic evaluation will be carried out to assess the performance of the optimized signage. Up to now, in some multi-directional wayfinding decision points which optimized signage has been constructed, the signage effectively guides and diverges passengers, and it is welcomed by the passengers according to monthly questionnaires.

The approach for data visualization based on HSB color code proposed in this paper provides a new perspective to analyze eye-tracking data. Taking the wayfinding decision point as the ordinate origin, three-dimensional coordinates of the interest area is established corresponding to the HSB value. It is helpful for us to directly analyze the relationship between the visual behavior, the signages, and the interior space. This method is helpful for researchers to make some assumptions and provides a definite direction for quantitative analysis of eye-tracking data. It can be used in the research of wayfinding, as well as environmental psychology and environmental behavior research.

(Note: all the charts in this paper are taken or drawn by the author).

Acknowledgements. The research was supported by a project of Natural Science Foundation of China titled "An internet-plus-based approach of crowd simulation for public buildings" (no. 51778417).

\section{References}

1. Arthur, P., Passini, R.: Wayfinding: People, Signs, and Architecture. McGraw-Hill, New York (1992)

2. Berger, C.: Wayfinding: Designing and Implementing Graphic Navigational Systems. Rockport Publishers, Beverly (2009)

3. Carpman, J.R., Grant, M.A.: Wayfinding: a broad view. In: Handbook of Environmental Psychology, pp. 427-442. Wiley, Washington (2002)

4. Kiefer, P., Giannopoulos, I., Raubal, M., Duchowski, A.: Eye tracking for spatial research: cognition, computation, challenges. Spat. Cognit. Comput. 17(1-2), 1-19 (2017)

5. Passini, R.: Wayfinding design: logic, application and some thoughts on universality. Des. Stud. 17(3), 319-331 (1996)

6. Schrom-Feiertag, H., Settgast, V., Seer, S.: Evaluation of indoor guidance systems using eye tracking in an immersive virtual environment. Spat. Cognit. Comput. 17(1-2), 163-183 (2017)

7. Sun, C., Yang, Y.: A study on visual saliency of way-finding landmarks based on eye-tracking experiments as exemplified in Harbin Kaide shopping center. Archit. J. 605(02), 24-29 (2019) 
8. Till, R.C., Babcock, J.S.: Proof of concept: use of eye-tracking to record how people use exit signage. In: Pedestrian and Evacuation Dynamics, pp. 209-219. Springer, Boston (2011). https://doi.org/10.1007/978-1-4419-9725-8_19

9. Tzeng, S.Y., Huang, J.S.: Spatial forms and signage in wayfinding decision points for hospital outpatient services. J. Asian Archit. Build. Eng. 8(2), 453-460 (2009)

10. Wiener, J.M., Hölscher, C., Büchner, S., Konieczny, L.: Gaze behaviour during space perception and spatial decision making. Psychol. Res. 76(6), 713-729 (2012)

11. Xu, L., Zhang, W., Tang, Z.: A virtue reality study of wayfinding and the sign layout in subway station. Archit. J. S1, 1-4 (2010)

Open Access This chapter is licensed under the terms of the Creative Commons Attribution 4.0 International License (http://creativecommons.org/licenses/by/4.0/), which permits use, sharing, adaptation, distribution and reproduction in any medium or format, as long as you give appropriate credit to the original author(s) and the source, provide a link to the Creative Commons license and indicate if changes were made.

The images or other third party material in this chapter are included in the chapter's Creative Commons license, unless indicated otherwise in a credit line to the material. If material is not included in the chapter's Creative Commons license and your intended use is not permitted by statutory regulation or exceeds the permitted use, you will need to obtain permission directly from the copyright holder.

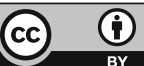

